\title{
ARTICLE
}

Cite this: DOI:

10.1039/x0xx00000x

\section{Mechanically and chemically robust ZIF-8 monoliths with high volumetric adsorption capacity}

\author{
Tian Tian, ${ }^{a}$ Jose Velazquez-Garcia, ${ }^{a}$ Thomas D. Bennett ${ }^{b}$ and David Fairen- \\ Jimenez ${ }^{a, *}$
}

Received $\odot \odot t h$ January 2012, Accepted ooth January 2012

DOI : $10.1039 / x \odot \times x \odot \odot \odot \odot \odot x$

WWW.rsc.org/
We report an easy synthetic procedure to produce large, crystalline, mechanically and chemically robust ZIF-8 monoliths without using any binders or high pressures. Gas adsorption studies show that the monolithic structures retain the characteristic porosity of ZIF-8 without any damage to the micropore system, while the bulk densities and volumetric BET areas are 3 times higher than the conventional, powder material. In addition, these structures are substantially more rigid than single crystals of the material.

\section{Introduction}

Zeolitic imidazolate frameworks (ZIFs), a sub-family of metalorganic frameworks (MOFs), are crystalline materials prepared by self-assembly of metal ions and imidazolate organic linkers. ${ }^{1}$ ZIFs adopt zeolitic topologies and display some of the quintessential stability of these classic inorganic materials. ${ }^{2}$ Their large pore volumes and surface areas, along with the possibility for chemical functionalization, have led to potential applications in gas adsorption, separation and catalysis.. ${ }^{3-8}$ However, the utility of ZIFs and MOFs in such applications is currently limited by an inability to process the microcrystalline powders resulting from their synthesis. Such shaping is very important in order to reduce the existence of pressure drops of a gas flow in columns due to powder compaction. In most cases, binders ${ }^{9}$ and/or high-pressure processes are used to pelletize the material, ${ }^{10}$ though often result in either i) partial or complete collapse of the internal porosity when using high pressures, ${ }^{11}$ or ii) pore blocking by the binder, preventing the access to the porosity. ${ }^{12}$ In addition, the use of a binder per se limits the amount of MOF in the final product, and hence would be expected to lead to reduced total guest capacities.

Despite of the rapid growth of MOF research, only few reports about the development of monolithic structures are available in the literature. In addition, the study of the mechanical properties of such MOF monolithic structures is generally overlooked. Most of the research on circumventing these problems concentrates on using high mechanical pressures for MOF densification in e.g. MOF- $177^{13}$ and MIL$101^{14}$, using pressures in the range of $0-10$ ton $\mathrm{cm}^{-2}$. However, even if these processes achieve high MOF densities, XRD analysis often suggests a gradual amorphization of the MOF when using high pressures, the collapse of internal pore structure and a resultant reduction of the adsorption capacity. HKUST-1 monoliths has been achieved by using a 2-step extrusion process, ${ }^{15}$ but the binders and additives used in this case resulted in a reduction of the porous solid fraction of the monolith and therefore a reduction in the adsorption capacity. Lohe et al. have previously prepared monolithic aerogels using the precursors from MIL-100, but the obtained materials resulted in very low densities, low volumetric capacities and an amorphous structure. ${ }^{16}$

In the current work, we focus mainly on ZIF-8 [ $\left.\mathrm{Zn}(\mathrm{mIm})_{2}\right]$ ( $\mathrm{mIm}=2$-methylimidazolate, $\mathrm{C}_{4} \mathrm{H}_{5} \mathrm{~N}_{2}{ }^{-}$), a well-known ZIF with sodalite topology. ${ }^{17-20}$ It contains large pore cavities (ca. $11.6 \AA$ diameter) interconnected by small windows (ca. $3.4 \AA$ diameter). Because ZIF-8 characteristic flexibility, these windows allow guest molecules larger than themselves into the porosity through a concerted swinging' motion of the $\mathrm{mIm}$ linkers. ${ }^{21,22}$ We describe here an easy and sequential method of producing large, mechanically and chemically robust ZIF-8 monoliths under ambient conditions without the use of binders, high pressures or high temperatures. The monoliths produced possess higher Young's moduli and hardness than single crystals of ZIF-8 and, crucially, retain the characteristic porosity of the framework - without any collapse of the micropore structure - while showing very high bulk densities. Furthermore, the resultant monoliths are transparent and fluorescence characteristics of previous ZIF-8 samples. The idea of using a sol-gel process to produce robust monolithic ZIF-8 is very generic, and so we were able to expand the process to ZIF-zni [ $\left.\mathrm{Zn}(\mathrm{Im})_{2}\right]$ ( $\mathrm{Im}=$ imidazolate, $\mathrm{C}_{3} \mathrm{H}_{3} \mathrm{~N}_{2}{ }^{-}$), a more dense ZIF material. ${ }^{23,24}$ 


\section{Experimental}

\section{Materials}

$\mathrm{Zn}\left(\mathrm{NO}_{3}\right) \cdot 6 \mathrm{H}_{2} \mathrm{O}$ (98\%), 2-methylimidazole (97\%), and imidazole (99\%) were purchased from Alfa Aesar; ethanol ( $\geq 99.5 \%$ ) and $\mathrm{NaOH}(10 \mathrm{M})$ were purchased from Sigma-Aldrich. The chemicals were used as received.

\section{Synthesis of ZIF-8 and ZIF-zni monoliths}

ZIF-8 synthesis was based on the method reported by Cravillon et al., ${ }^{19}$ Solutions of 2-methylimidazole $(20 \mathrm{ml}, 0.395 \mathrm{M})$ and $\mathrm{Zn}\left(\mathrm{NO}_{3}\right) \cdot 6 \mathrm{H}_{2} \mathrm{O}(20 \mathrm{ml}, 0.049 \mathrm{M})$ in ethanol were mixed and stirred for 2 hours at room temperature. After centrifugation, the collected solid was washed in ethanol (20 ml, 3 times) under ultrasonication for 3 minutes. The collected solid was processed by four different methods. First, ZIF-8HT (i.e. HT, high temperature) was obtained by drying a fraction of the white solid at $100{ }^{\circ} \mathrm{C}$ overnight in a vacuum oven. Second, ZIF-8LT (i.e. LT, low temperature) was obtained by drying a second fraction of the white powder at room temperature overnight. Third, ZIF-8LT-HT was obtained by further evacuation of ZIF-8LT at $100{ }^{\circ} \mathrm{C}$ overnight in a vacuum oven. Finally, ZIF-8ER (i.e. extended reaction) was obtained by washing the initial white solid twice in ethanol and then dispersed in $40 \mathrm{ml}$ of ethanol. 2methylimidazole $(0.395 \mathrm{M})$ and $\mathrm{Zn}\left(\mathrm{NO}_{3}\right) \cdot 6 \mathrm{H}_{2} \mathrm{O}(0.049 \mathrm{M})$ were then added to the solution and the mixture was ultrasonicated for 10 minutes at room temperature and centrifuged. The collected solid was washed in ethanol (20 ml, 3 times) under ultrasonication for 3 minutes and dried at room temperature overnight. The resulting monoliths, with sizes ranging $1 \mathrm{~mm}^{3}$ to $1 \mathrm{~cm}^{3}$ are shaped according to the mould used during the drying process.

ZIF-zni was synthesised by a similar method. $1 \mathrm{ml}$ of $\mathrm{NaOH}(1 \mathrm{M})$ was added to a solution of imidazole $(20 \mathrm{ml}, 0.395 \mathrm{M})$ in ethanol. This was mixed with a solution of $\mathrm{Zn}\left(\mathrm{NO}_{3}\right) \cdot 6 \mathrm{H}_{2} \mathrm{O}(20 \mathrm{ml}, 0.049 \mathrm{M})$ in ethanol, and stirred for 2 hours at room temperature. After centrifugation at $5500 \mathrm{rpm}$ for 10 minutes, a white solid was collected and dried at room temperature overnight.

\section{Characterisation of the materials}

Powder X-ray diffraction (XRD) patterns were recorded with a Bruker D8 diffractometer using $\mathrm{CuK} \alpha_{1}(\lambda=0.15405 \AA)$ radiation with a step of $0.02^{\circ}$ at a scanning speed of $0.1^{\circ} \mathrm{s}^{-1}$. Scanning electron microscope (SEM) images were taken using a FEI XL30 FEGSEM with an accelerating voltage of $5 \mathrm{kV}$. Transmission electron microscopy (TEM) images were obtained using a FEI Tecnai G2 with a $200 \mathrm{kV}$ voltage. For the TEM images, $1 \mathrm{ml}$ of mother solution was taken and diluted 10 times in ethanol before centrifugation. Then, $50 \mu$ of the solution was dripped on a copper grid and the TEM image was taken after the ethanol was evaporated at room temperature. Thermogravimetric analysis (TGA) was performed using a Pyris 1 TGA, from room temperature to $750{ }^{\circ} \mathrm{C}$, using a ramp rate of $10{ }^{\circ} \mathrm{C} \mathrm{min}^{-1}$. $\mathrm{N}_{2}$ adsorption isotherms were undertaken at $77 \mathrm{~K}$ using a Micromeritics ASAP 2020 instrument. Mercury porosimetry was obtained up to a final pressure of 2000 bar using an AutoPore IV 9500 instrument. With this technique, the volume of the pores greater than $100 \mathrm{~nm}$ and the bulk particle density at atmospheric pressure were obtained. Prior to the $\mathrm{N}_{2}$ adsorption and the mercury porosimetry analysis, all samples were evacuated overnight for $24 \mathrm{~h}$ at $150{ }^{\circ} \mathrm{C}$ under vacuum.

\section{Nanoindentation}

Nanoindentation experiments were performed using an MTS Nanoindenter XP, located in an isolation cabinet to shield against thermal fluctuations and acoustic interference. Before indentation, monolith surfaces were first cold-mounted using an epoxy resin and then carefully polished using increasingly fine diamond suspensions. Indentations were conducted under the dynamic displacementcontrolled "continuous stiffness measurement" mode. E (Elastic modulus) and $H$ (Hardness) mechanical properties were subsequently determined as a function of the surface penetration depth. A 2-nm sinusoidal displacement at $45 \mathrm{~Hz}$ was superimposed onto the system's primary loading signal, and the loading and unloading strain rates were set at $5 \times 10^{-2} \mathrm{~s}^{-1}$. All tests were performed to a maximum indentation depth of $1,000 \mathrm{~nm}$ using a Berkovich (i.e. three-sided pyramidal) diamond tip of radius $\sim 100$ $\mathrm{nm}$. The raw data (load-displacement curves) obtained were analysed using the Oliver and Pharr method, ${ }^{25}$ and Poisson's ratio set at 0.2 , in accordance with prior work on zeolitic imidazolate frameworks. ${ }^{26}$ Data resulting from surface penetrations of less than $100 \mathrm{~nm}$ were discarded due to imperfect tip-surface contacts.

\section{Chemical stability tests}

The stability of ZIF-8ER was tested in refluxing water at $100{ }^{\circ} \mathrm{C}$ for seven days. The stability was monitored using XRD every 48 hours from day 3.

\section{Results and discussion}

Figures 1a and b show images of ZIF-8HT and ZIF-8LT, respectively. While the white pellets of ZIF-8HT easily disaggregate into a typical white ZIF-8 powder, both ZIF-8LT and ZIF-8LT-HT remain as transparent monolithic structures.

The retention of the macroscopic monolithic morphology of ZIF-8LT during higher temperature activation is remarkable. Figure 1d shows the fluorescence of ZIF-8LT. Its transparent nature makes the material a perfect candidate for sensing applications since the fluorescence will be reproduced in the bulk solid. Figure 2 shows the PXRD pattern of the different samples. The three samples are identical in crystalline structure, despite the differences in their morphologies, which were investigated using SEM. Figure 3 shows that ZIF-8HT presents a significant amount of interstitial spaces between primary particles, associated to pores in the range of the meso- and macroporosity, whereas ZIF-8LT and ZIF-8LT-HT present a relatively flat surface. The different morphologies at the macroand micro-scale resemble those previously observed for xerogels and aerogels, ${ }^{16,27}$ not only for amorphous MOF-like materials ${ }^{14}$ but also for carbon and silica aerogels ${ }^{28}$. Primary particle sizes of ZIF-8 from the initial mixture of precursors, obtained by TEM, were around 60-70 nm (Figure S1). 

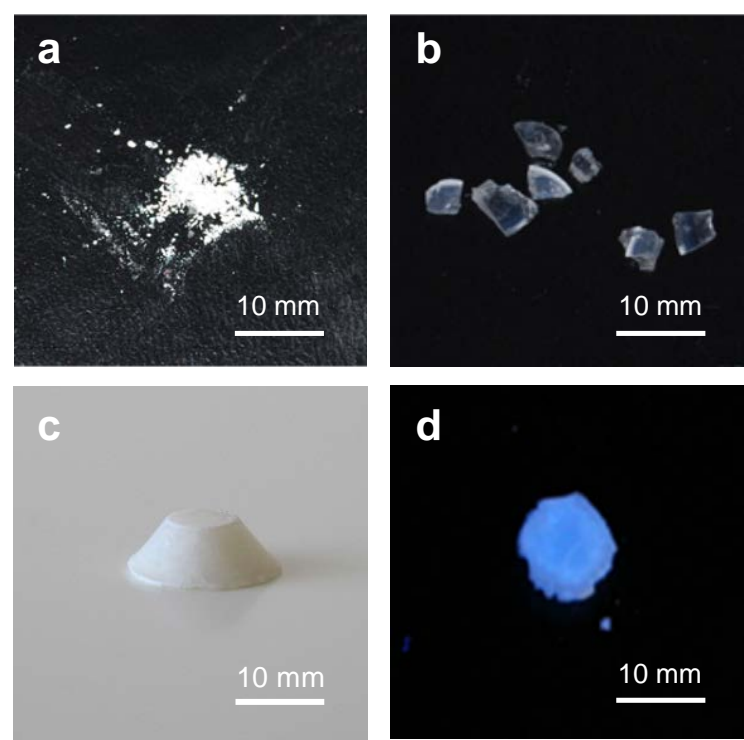

Fig. 1. Optical pictures of a) ZIF-8HT;b) ZIF-8LT; c) ZIF-8ER and d) ZIF-8ER, under $365 \mathrm{~nm}$ UV light. e) PXRD patterns of the different samples alongside a simulated pattern for ZIF-8.

The formation of the monolithic structures stems from the existence of small primary particles and the mild drying conditions. The fact that ZIF-8LT and ZIF-8LT-HT are transparent and therefore do not show visible light scattering is presumably related to the absence of electronic contrast between phases ${ }^{29}$ or the existence of primary particles smaller than the light wavelength. ${ }^{30}$ At this point, we hypothesise that the existence of positively charged ZIF-8 particles $^{19}$ and residuary reactants ( $\mathrm{mIm}$ and $\mathrm{Zn}$ ions) within the sample and the mild drying process allows extension of the polymerisation reaction and the formation of the monolithic structure. In this case, new ZIF-8 is formed during the drying process of ZIF$8 \mathrm{LT}$ at room temperature, acting as a binder of the primary ZIF8 particles. To investigate this hypothesis, we proceeded with

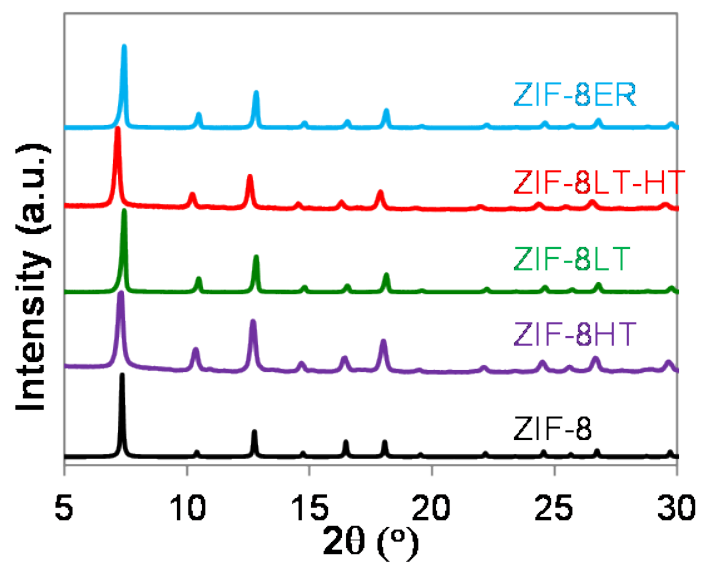

Fig. 2 PXRD patterns of the different samples alongside a simulated pattern for ZIF-8.
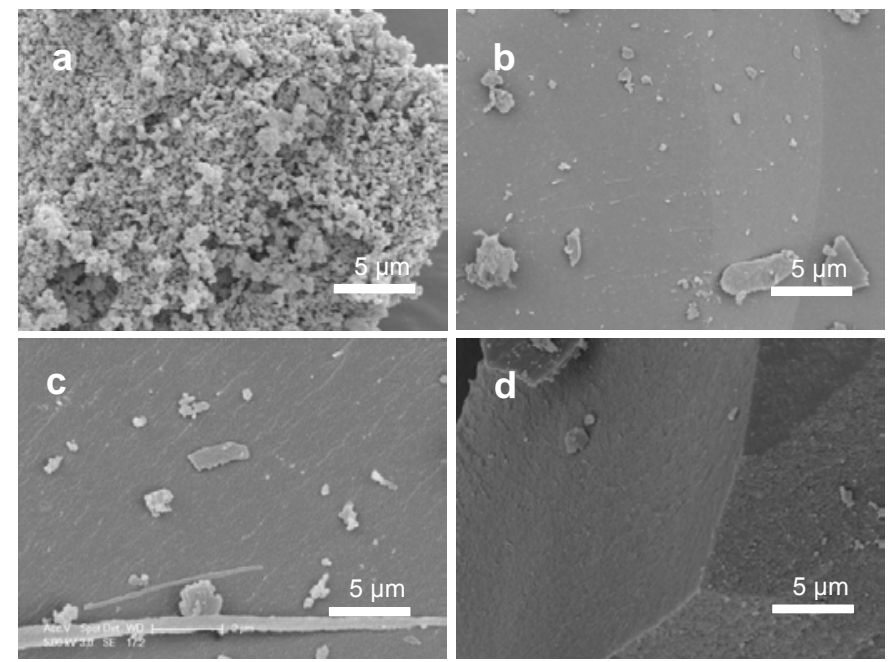

Fig. 3. SEM images of a) ZIF-8HT, b) ZIF-8LT, c) ZIF-8LT-HT and d) ZIF-8 ER.

the synthesis of a new sample, where the initial ZIF-8 precipitate, was dispersed in ethanol immediately after centrifugation and included in a new solution of $\mathrm{mIm}$ and $\mathrm{Zn}\left(\mathrm{NO}_{3}\right) \cdot 6 \mathrm{H}_{2} \mathrm{O}$. This mixture was ultrasonicated for 10 minutes at room temperature, centrifuged at $5500 \mathrm{rpm}$, washed and dried at room temperature overnight. We named the resulting white monolithic structure from the extended-reaction sample as ZIF-8ER. Figure 1c shows the picture for its macroscopic monolithic structure, whereas Figure 2 and Figure 3d show the PXRD pattern and SEM image respectively. Figure S2 shows that no preferential orientation of ZIF-8LT and ZIF-8LT-HT was observed, with X-ray diffraction patterns of powder and bulk pieces being identical.

We analysed the porosity of the prepared samples by using $\mathrm{N}_{2}$ adsorption at $77 \mathrm{~K}$. Figure $4 \mathrm{a}$ and $\mathrm{S} 3$ show the results of gravimetric capacity in a semi-logarithmic and linear scale, respectively. Table 1 and $\mathrm{S} 1$ show the main analysis results. All the samples presented the typical structural flexibility and stepwise adsorption mechanism of $\mathrm{N}_{2}$ in ZIF-8, ${ }^{31}$ which indicates the samples were indeed microporous (porosity diameter $<2$ $\mathrm{nm}$ ). In addition, ZIF-8HT showed a large increase in the $\mathrm{N}_{2}$ adsorption at higher pressures, close to saturation pressure. This large increase is related to $\mathrm{N}_{2}$ condensation in the wider porosity existing in the sample, which is consistent with the existence of meso- and macroporosity from interstitial spaces between particles observed in the SEM. The gravimetric BET areas of the monolithic materials were around $1390 \mathrm{~m}^{2} / \mathrm{g}$, whereas gravimetric BET areas of conventional, powder ZIF-8 are generally in the range of $1300-1600 \mathrm{~m}^{2} / \mathrm{g},{ }^{32}$ meaning that the monolithic materials retained the characteristic porosity of ZIF-8. Moreover, volumetric adsorption capacities (i.e. the amount of gas that can be adsorbed per $\mathrm{cm}^{3}$ of a specific material) and volumetric BET areas, which can be obtained by 


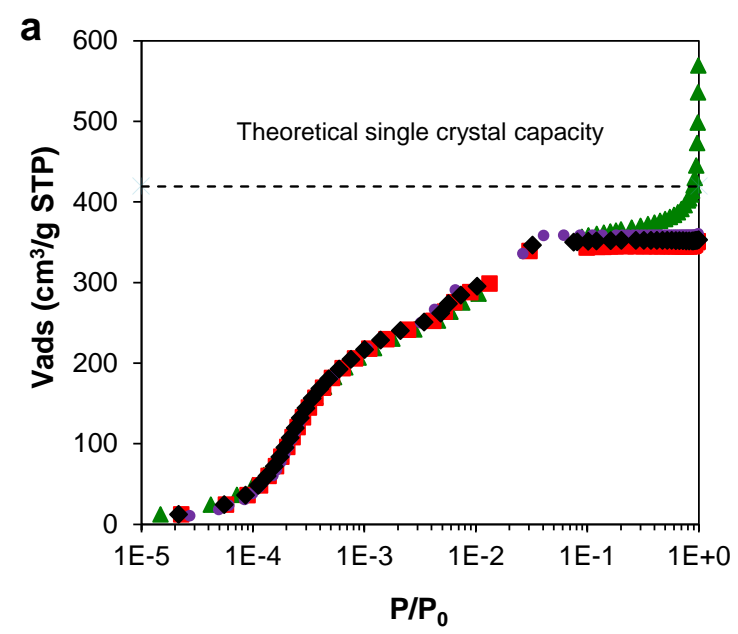

b

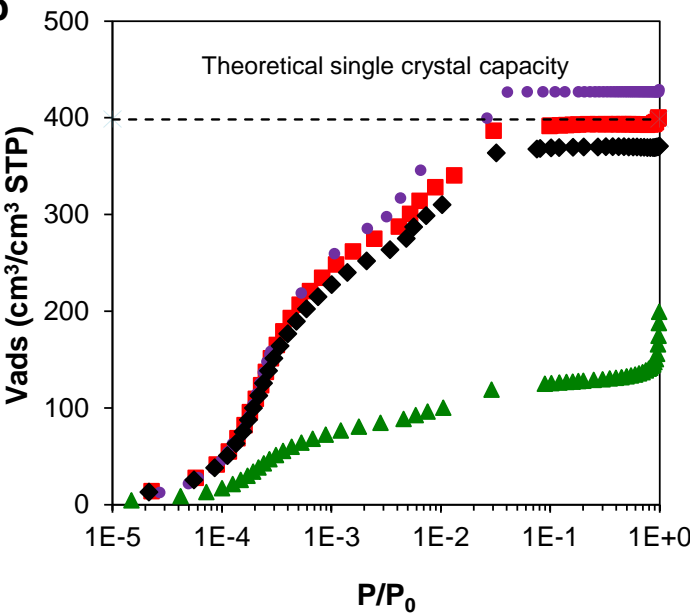

Fig. 4 a) Gravimetric and b) volumetric $\mathrm{N}_{2}$ adsorption isotherms at 77 $\mathrm{K}$ for ZIF-8LT, red squares, ZIF-8HT, green triangles, ZIF-8LT-HT, black diamonds, and ZIF-8ER, purple circles. Dotted black line shows the theoretical single-crystal adsorption capacity. Note the use of semilogarithmic scale to get more detail in the low pressure range.

multiplying gravimetric data with bulk density of the sample, are especially important from an applied point of view in most industrial applications when the adsorbent material has to be confined in a fixed given volume.

In order to calculate the volumetric capacities, we measured the density of the samples using mercury porosimetry in the activated samples (Figure S4). Since mercury does not penetrate the porous system of the materials at atmospheric pressure, it allows measuring the total bulk volume of the samples by applying Archimedes' method, which in turn facilitates calculation of their bulk densities (Table 1 and S1). ${ }^{16 \text {, }}$ ${ }^{28}$ Table 1 and S1 show the results. Although the crystal density of ZIF-8 is high (ca. $0.95 \mathrm{~g} / \mathrm{cm}^{3}$ ), it does not include interparticle spaces that eventually will reduce the bulk density of the powder down to $c a .50 \%$ of the actual value. ${ }^{33}$ Indeed, commercial ZIF-8 from BASF ${ }^{34}$ presents a bulk density of 0.35 $\mathrm{g} / \mathrm{cm}^{3}$. In contrast, all the monolithic structures revealed outstanding high bulk densities. The fact that measured densities are higher than crystal density of ZIF-8 might be
Table 1. BET areas $\left(\mathrm{S}_{\mathrm{BET}}\right)$, micropore volume $\left(\mathrm{W}_{0}\right)$, total pore volume $\left(\mathrm{V}_{\mathrm{TOt}}\right)$ and bulk density $(\rho b)$ for the different ZIF-8 structures.

\begin{tabular}{ccccccc} 
Material & $\begin{array}{c}\mathrm{S}_{\mathrm{BET}} \\
\mathrm{m}^{2} / \mathrm{g}\end{array}$ & $\begin{array}{c}\mathrm{W}_{0}{ }^{\mathrm{a}} \\
\mathrm{cm}^{3} / \mathrm{g}\end{array}$ & $\begin{array}{c}\mathrm{V}_{\text {Tot }} \mathrm{b}^{3} / \mathrm{g} \\
\mathrm{cm}^{3}\end{array}$ & $\begin{array}{c}\rho_{\mathrm{b}}{ }^{\mathrm{b}} \\
\mathrm{g}^{3} \mathrm{~cm}^{\mathrm{d}}\end{array}$ & $\begin{array}{c}\mathrm{S}_{\mathrm{BET}}(\mathrm{vol}) \\
\mathrm{m}^{2} / \mathrm{cm}^{3}\end{array}$ & $\begin{array}{c}\mathrm{W}_{\mathrm{o}}(\mathrm{vol}) \\
\mathrm{cm}^{3} / \mathrm{cm}^{3}\end{array}$ \\
\hline ZIF-8HT & 1387 & 0.552 & 0.829 & $0.35^{\mathrm{d}}$ & 485 & 0.193 \\
ZIF-8LT & 1359 & 0.532 & 0.543 & 1.14 & 1549 & 0.606 \\
ZIF-8LT-HT & 1423 & 0.543 & 0.546 & 1.05 & 1494 & 0.570 \\
ZIF-8ER & 1395 & 0.535 & 0.545 & 1.19 & 1660 & 0.637 \\
\hline
\end{tabular}

${ }^{\mathrm{a}}$ Obtained at $\mathrm{P} / \mathrm{P}_{0}=0.1 ;{ }^{\mathrm{b}}$ Obtained at $\mathrm{P} / \mathrm{P}_{0}=0.99 ;{ }^{\mathrm{c}}$ Bulk density quantified by measurement of weight and volume using mercury porosimetry. ${ }^{\mathrm{d}}$ ZIF-8 density as reported by BASF.

explained by the presence of impurities or a non-complete activation. This is probably the reason of the slightly lower gravimetric surface areas.

Figure $4 \mathrm{~b}$ and S3c show the volumetric adsorption isotherms of ZIF-8 monoliths with powder ZIF-8. The effect of the density differences between samples on the volumetric adsorption is very significant. First, the low density of powder ZIF-8 means that the volumetric capacity, BET area and micropore volume are very low. Then, the monolithic materials prepared here present an outstanding enhancement of the conventional, powder ZIF-8, with values more than 3 times higher: 1660 vs. $485 \mathrm{~m}^{2} / \mathrm{cm}^{3}$ for ZIF-8ER and powder ZIF-8, respectively, due to the high densities. The fact that the volumetric adsorption capacity is higher than the theoretical single crystal capacity, which is calculated from the $18 \mathrm{mmol} / \mathrm{g}$ $\mathrm{N}_{2}$ capacity and a crystal density of $0.95 \mathrm{~g} / \mathrm{cm}^{3}$ could be related to the existence of issues when calculating bulk densities or the existence of impurities. Efforts towards MOF densification
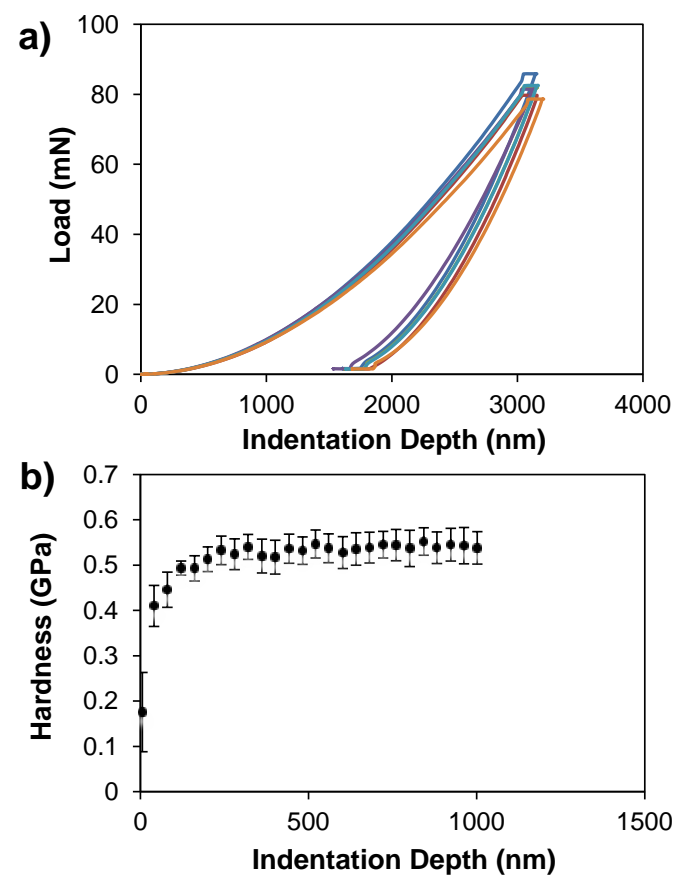

Fig. 5 a) Load-displacement data for 6 indents and b) hardness as a function of indentation depth on ZIF-8ER monoliths. 
Table 2. Mechanical properties of different ZIF-8 structures. Single crystal data has been obtained in the $\{1,0,0\}$ facet.

\begin{tabular}{ccc} 
Material & $\begin{array}{c}\text { Elastic Modulus, } E \\
\text { GPa }\end{array}$ & $\begin{array}{c}\text { Hardness, } H \\
\text { GPa }\end{array}$ \\
\hline ZIF-8 single crystal $^{26}$ & $2.973 \pm 0.05$ & $0.501 \pm 0.023$ \\
ZIF-8LT & $3.66 \pm 0.18$ & $0.417 \pm 0.038$ \\
ZIF-8LT-HT & $3.57 \pm 0.22$ & $0.429 \pm 0.026$ \\
ZIF-8ER & $7.04 \pm 0.13$ & $0.643 \pm 0.021$ \\
\hline
\end{tabular}

have been addressed before for MOF- $177,{ }^{13}$ where the density of MOF-177 increased from $0.1 \mathrm{~g} / \mathrm{cm}^{3}$ up to $1.40 \mathrm{~g} / \mathrm{cm}^{3}$. However, in all the cases the volumetric capacities were below the theoretical single crystal capacities. The fact that the maximum volumetric capacity for MOF-177 was obtained for pellets with a density of $0.53 \mathrm{~g} / \mathrm{cm}^{3}$ before decreasing for higher preparation pressures suggest a gradual amorphization when using higher pressures, causing the collapse of the porosity. This was indeed confirmed by XRD studies on the pellets obtained at very high pressures.

In order to use MOF-monoliths in e.g. column beds or fuel tanks, they must have appropriate mechanical properties to support mechanical stresses, which come from the weight of the adsorbent inside the columns and from vibrations or movements of the bed. To evaluate the mechanical properties of the obtained monoliths, we studied the Young's modulus, E, and hardness, $H$, by nanoindentation, based on the dynamic mode load-displacement data (Figures 5 and S5-S20). Table 2 shows comparable $\mathrm{H}$ values to those seen before, though Young's moduli were significantly higher. ${ }^{26}$ Measurements could only be performed on one face of the monoliths because of the small area available on others. Preferential orientation of ZIF-8 monoliths was not observed (Figure S1). As can be seen in Table 2, the elastic modulus of ZIF-8ER is significantly higher than the previous monoliths and the ZIF-8 single crystal.

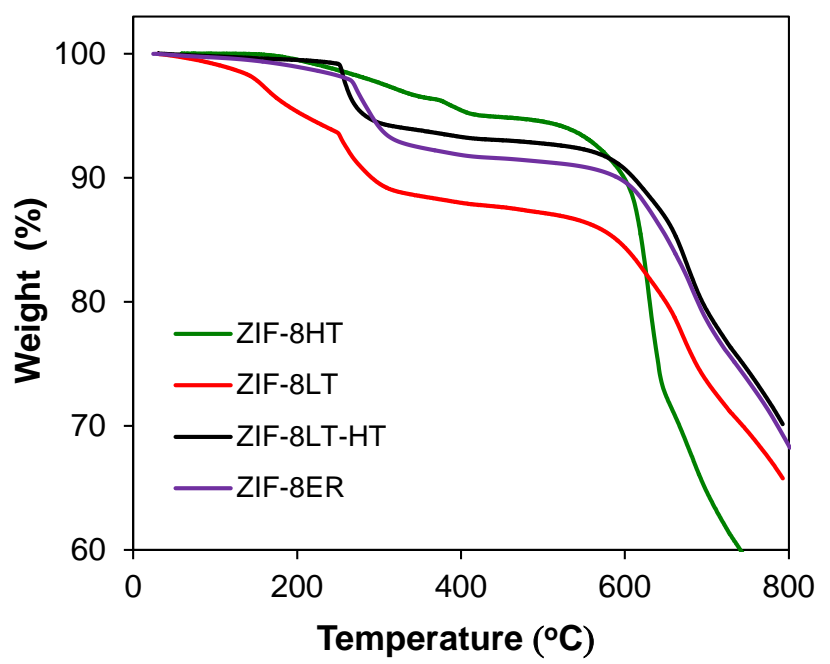

Fig. 6. Thermogravimetric analysis of the different ZIF-8 samples.

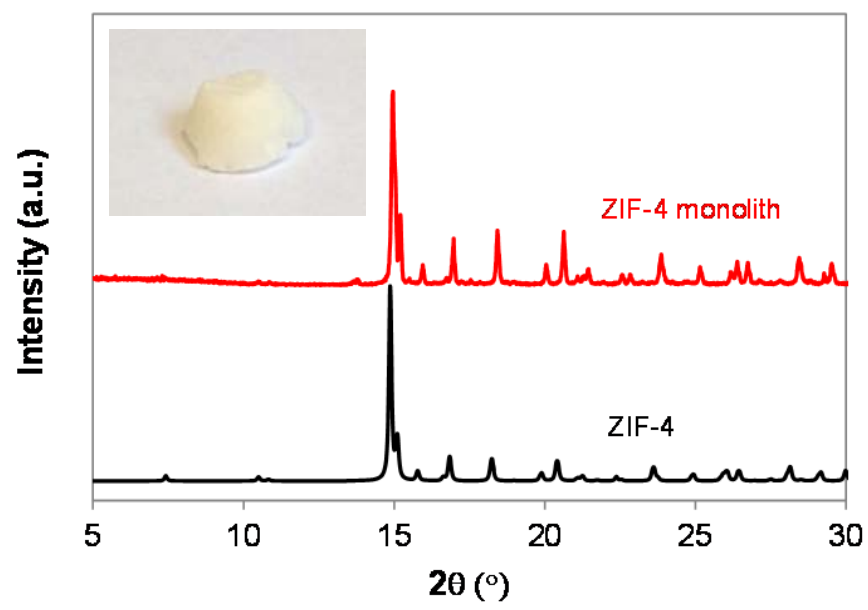

Fig. 7. PXRD patterns of the ZIF-zni monolith alongside a simulated pattern for ZIF-zni. The inset depicts an optical picture of the ZIF-zni monolithic structure.

The high values of $E$ reported can be compared to thin films of ZIF-8 (3.5 GPa) prepared by Eslava et al., ${ }^{35}$ where the deviation in moduli from single crystals was assigned to surface roughness effects. Notable differences in $E$ between thin films and single-crystals of HKUST- $1^{36}$ (9.3 and $3.5 \mathrm{GPa}$, respectively) have been noted before and ascribed to elastic anisotropic effects. We also studied the stability of the samples by using thermogravimetric analysis (TGA). Figure 6 shows sharp weight losses of 12,7 and $8 \%$ up to $300{ }^{\circ} \mathrm{C}$ for ZIF-8LT, ZIF-8LT-HT and ZIF-8ER respectively. These losses are attributed to the residual ethanol and water in the materials. ZIF-8HT shows a weight loss of $\mathrm{ca}$. $4 \%$ up to $300{ }^{\circ} \mathrm{C}$, indicating that the residual solvent molecules in powders might be easier to be removed than in monoliths. A second weight loss step due to thermal degradation is observed at $600{ }^{\circ} \mathrm{C}$ for all three samples, which is consistent with the previous literature reports. ${ }^{37}$

The stability of the monolithic ZIF-8ER was tested in water at $100{ }^{\circ} \mathrm{C}$. for 7 days Figure S21 shows the PXRD patterns of the samples at 3, 5 and 7 days. After being immersed in boiling water, ZIF-8ER was able to keep the monolithic morphology and the crystalline structure of ZIF-8, similar to previous reported data for standard, powder ZIF-8. ${ }^{1,37}$

In order to expand the method to other materials, we switched the organic linker from 2-methylimidazole to imidazole. During the synthesis, $1 \mathrm{ml}$ of $\mathrm{NaOH}$ was added in order to improve the deprotonation of the organic linker. In this case, we obtained identical monolithic morphologies to those of ZIF-8. Figure 7 shows the PXRD pattern of the monolith, confirming that the structure was ZIF-zni, the densest of the known $\mathrm{Zn}(\mathrm{Im})_{2}$ phases.

\section{Conclusion}

We have synthesised transparent, robust monoliths of ZIF-8 by a simple process, which is a low-cost strategy to create MOF 
monoliths without using binders and/or high pressures. Monolithic materials retained the characteristic porosity of ZIF8 while showing bulk densities and volumetric BET areas three times higher than the conventional, powder material. In addition, samples were substantially more rigid than single crystals of ZIF-8.

We have shown that the existence of ZIF-8 precursors in the solution as well as a slow drying process is key to obtain a mechanically robust monolith: i) when the drying process is fast (at high temperature, ZIF-8HT), the sample is a powder; ii) when the drying process is slow (at room temperature, ZIF8LT), the sample is a monolith; iii) when higher amounts of precursor are included in the second step and the drying is slow (ZIF-8ER), the sample is a monolith with superior mechanical properties. On one hand, a slow drying process allows time for the reaction of the precursors. On the other hand, a mild, slow drying process will reduce the stress around the vapour-liquid meniscus during the evaporation of the solvent found in the interstitial spaces between primary ZIF-8 particles

We have also extended this method to synthesise ZIF-zni monolith, which provides the possibility to expand the approach to other monolithic-MOF synthesis. All these make the reported process ideal for industrial applications of MOFs where optimal materials need to present high volumetric adsorption capacities and high mechanical properties.

\section{Acknowledgements}

This work was funded by the EPSRC IAA Partnership Development Award (RG/75759). D.F.-J. thanks the Royal Society for funding through a University Research Fellowship. T.D.B would like to thank Trinity Hall for funding and Professor Anthony Cheetham for use of lab facilities and equipment.

\section{Notes and references}

${ }^{a}$ Department of Chemical Engineering \& Biotechnology, University of Cambridge, Pembroke Street, Cambridge CB2 3RA, United Kingdom. Email: df334@cam.ac.uk; website: http://people.ds.cam.ac.uk/df334

${ }^{b}$ Department of Materials Science and Metallurgy, University of Cambridge, 27 Charles Babbage Road, Cambridge CB3 OFS, United Kingdom

†Electronic Supplementary Information (ESI) available: PXRD patterns, transmission electron microscopy, nanoindentation, gas adsorption, mercury porosimetry. See DOI: 10.1039/b000000x/.

1. K. S. Park, Z. Ni, A. P. Côté, J. Y. Choi, R. Huang, F. J. Uribe-Romo, H. K. Chae, M. O'Keeffe, and O. M. Yaghi, Proc. Natl. Acad. Sci. U. S. A., 2006, 103, 10186.

2. Y.Q. Tian, Y.M. Zhao, Z.X. Chen, G.N. Zhang, L.H. Weng, and D.Y. Zhao, Chem. Eur. J., 2007, 13, 4146.

3. H. Furukawa, K. E. Cordova, M. O’Keeffe, and O. M. Yaghi, Science, 2013, 341, 1230444.

4. T. D. Bennett, P. J. Saines, D. A. Keen, J.-C. Tan, and A. K. Cheetham, Chem. Eur. J., 2013, 19, 7049.

5. Y. He, W. Zhou, G. Qian, and B. Chen, Chem. Soc. Rev., 2014, 43, 5657.

6. K. Zhang, R. P. Lively, M. E. Dose, A. J. Brown, C. Zhang, J. Chung, S. Nair, W. J. Koros, and R. R. Chance, Chem. Commun., 2013, 49, 3245.
7. E. Barea, C. Montoro, and J. A. R. Navarro, Chem. Soc. Rev., 2014, 43, 5419.

8. J. Liu, L. Chen, H. Cui, J. Zhang, L. Zhang, and C.-Y. Su, Chem. Soc. Rev., 2014, 6011.

9. C. A. Grande, V. I. Águeda, A. Spjelkavik, and R. Blom, Chem. Eng. Sci., 2014, 1.

10. D. Bazer-Bachi, L. Assié, V. Lecocq, B. Harbuzaru, and V. Falk, Powder Technol., 2014, 255, 52.

11. K. W. Chapman, G. J. Halder, and P. J. Chupas, J. Am. Chem. Soc., 2009, 131, 17546.

12. V. Finsy, L. Ma, L. Alaerts, D. E. De Vos, G. V. Baron, and J. F. M. Denayer, Micropor. Mesopor. Mat., 2009, 120, 221.

13. R. Zacharia, D. Cossement, L. Lafi, and R. Chahine, J. Mater. Chem., 2010, 20, 2145.

14. H. Oh, D. Lupu, G. Blanita, and M. Hirscher, RSC Adv., 2014, 4, 2648.

15. P. Küsgens, A. Zgaverdea, H.G. Fritz, S. Siegle, and S. Kaskel, J. Am. Ceram. Soc., 2010, 93, 2476.

16. M. R. Lohe, M. Rose, and S. Kaskel, Chem. Commun. 2009, 6056.

17. X.-C. Huang, Y.-Y. Lin, J.-P. Zhang, and X.-M. Chen, Angew. Chem. Int. Ed., 2006, 45, 1557.

18. S. R. Venna, J. B. Jasinski, and M. A. Carreon, J. Am. Chem. Soc., 2010, 132, 18030.

19. J. Cravillon, S. Münzer, S.-J. Lohmeier, A. Feldhoff, K. Huber, and M. Wiebcke, Chem. Mater., 2009, 21, 1410.

20. J. Cravillon, R. Nayuk, S. Springer, A. Feldho, K. Huber, and M. Wiebcke, Chem. Mater., 2011, 2130.

21. S. A. Moggach, T. D. Bennett, and A. K. Cheetham, Angew. Chem. Int. Ed., 2009, 48, 7087.

22. D. Fairen-Jimenez, S. A. Moggach, M. T. Wharmby, P. A. Wright, S. Parsons, and T. Düren, J. Am. Chem. Soc., 2011, 133, 8900.

23. R. Lehnert, F. Seel, Z. Anorg. Allg. Chem., 1980, 464, 187.

24. T. D. Bennett, A. L. Goodwin, M. T. Dove, D. A. Keen, M. G. Tucker, E. R. Barney, A. K. Soper, E. G. Bithell, J. Tan, and A. K. Cheetham, Phys. Rev. Lett., 2010, 104, 115503.

25. W. C. Oliver and G. M. Pharr, J Mater Res, 2004, 19, 3.

26. J. C. Tan, T. D. Bennett, and A. K. Cheetham, Proc. Natl. Acad. Sci. U. S. A., 2010, 107, 9938.

27. L. Li, S. Xiang, S. Cao, J. Zhang, G. Ouyang, L. Chen, and C.Y. Su, Nat. Commun., 2013, 4, 1774.

28. D. Fairén-Jiménez, F. Carrasco-Marín, and C. Moreno-Castilla, Langmuir, 2008, 24, 2820.

29. D. Fairén-Jiménez, F. Carrasco-Marín, D. Djurado, F. Bley, F. EhrburgerDolle, and C. Moreno-Castilla, J. Phys. Chem. B, 2006, 110, 8681.

30. R. Apetz and M. P. B. Bruggen, J. Am. Ceram. Soc., 2003, 86, 480.

31. D. Fairen-Jimenez, R. Galvelis, A. Torrisi, A. D. Gellan, M. T. Wharmby, P. A. Wright, C. Mellot-Draznieks, and T. Düren, Dalton Trans., 2012, 41, 10752

32. Q. Song, S. K. Nataraj, M. V. Roussenova, J. C. Tan, D. J. Hughes, W. Li, P. Bourgoin, M. A. Alam, A. K. Cheetham, S. a. Al-Muhtaseb, and E. Sivaniah, Energy Environ. Sci., 2012, 5, 835.

33. J. Juan-Juan, J. P. Marco-Lozar, F. Suárez-García, D. Cazorla-Amorós, and a. Linares-Solano, Carbon, 2010, 48, 2906.

34. Sigma-Aldrich, http://www.sigmaaldrich.com/catalog/product/aldrich/691348?lang=en\&r egion=GB, (accessed Jun 2014).

35. S. Eslava, L. Zhang, S. Esconjauregui, J. Yang, K. Vanstreels, M. R. Baklanov, and E. Saiz, Chem. Mater., 2013, 25, 27.

36. S. Bundschuh, O. Kraft, H. K. Arslan, H. Gliemann, P. G. Weidler, and C. Wöll, Appl. Phys. Lett., 2012, 101, 101910.

37. Y. Pan, Y. Liu, G. Zeng, L. Zhao, and Z. Lai, Chem. Commun., 2011, 47, 2071. 